\title{
VR Interactive Feature of HTML5-based WebVR Control Laboratory by Using Head-mounted Display
}

\author{
https://doi.org/10.3991/ijoe.v14i03.8112 \\ Quan Ye, Wenshan $\left.\mathrm{Hu}^{(}{ }^{\bowtie}\right)$, Hong Zhou, Zhongcheng Lei, Shiqi Guan \\ Wuhan University, Wuhan, China \\ wenshan.hu@whu.edu.cn
}

\begin{abstract}
The development of web-based online laboratory for engineering education has made big progress in recent years with state-of-the-art technologies. In order to further promote the effectiveness within an online virtual environment, the virtual reality (VR) interactive feature of WebVR Control laboratory based on HTML5 is presented in this paper. The educational objective is to assist the students or researchers to conduct a realistic and immersive experiment of control engineering with a head-mounted display (HMD) equipped. The implementation of the VR technique mainly relies on the Three.js graphic engine based on Web Graphics Library (WebGL) which has been supported by most of the mainstream web browsers in their latest version without a plugin embedded. At the end of the paper, the ball and beam system is taken as an example to illustrate the whole operational approach of the VR experiment in detail. While such a VR interactive feature hasn't been integrated into some of the current web-based online laboratories, the technical solution could be inspirational for them.
\end{abstract}

Keywords-HTML5, WebVR, virtual reality, head-mounted display, control engineering education, online laboratory

\section{Introduction}

In recent years, online laboratory is widely proposed and recognized as a significant technology to support life-long education and students' autonomous learning activities along with a flexible schedule. As summarized and analyzed in [1], [2], a number of hybrid laboratories [3] (the combination of both remote laboratories and virtual laboratories) in the realm of control engineering education have been established all over the world both for formal-institutional education and informal massive education. While traditional hands-on laboratories often involve high costs associated with equipment, space, and maintenance staff [3], hybrid laboratories can serve as cost-effective alternatives to conventional education and research tools. Moreover, hybrid laboratories provide additional advantages in aspects of availability, observability, accessibility and safety [4]. In [1], [5], [6], [7], authors all give pertinent reviews that student learning outcome achievement is equal or higher in hybrid laboratories versus traditional laboratories. 
As one of the well-developed hybrid laboratories, Networked Control System Laboratory (NCSLab) [8]-[13] experiences intensive development over the past decade. New features and new technologies were gradually applied into the NCSLab framework. More specifically, 3D animation synchronized with remote experiments was added in 2013 as illustrated in [11]. Three years later, [12] presents a HTML5 version of NCSLab which provides better user experience with fluent 3D animation in experimental process. And the efforts of the NCSLab team toward a powerful hybrid laboratory to meet the engineering education's requirements continue up to now.

In order to provide highly realistic and immersive ambience for users in virtual experimental process, many hybrid laboratories have made effort in integrating various form of interactive mode based on state-of-the-art technologies and standards, one of which is the virtual reality (VR). The concept of virtual reality refers to the combination of computer graphics systems with various displays and interface devices to provide the effect of immersion in the interactive $3 \mathrm{D}$ computer-generated environment [14], [15]. Burdea Grigore and Coiffet concluded the characteristics of VR with 3I (immersion, interaction and imagination) in [16].

The VR technology has long attracted public's attention since its advent in 1960's, especially in the education realm. Though it is a globally focused hotspot, VR, whose wide spread dissemination is restricted by technical concerns [17], poor instructional design [18] and lack of solid hardware infrastructure, deed experienced a downturn for some time in the early $21^{\text {st }}$ century. While the 2016 releases of the Oculus Rift CV1, HTC Vive, and the years earlier release of the Google Cardboard, spawned a frenzy of VR again with explosive growth of VR headset shipments.

The NMC Horizon Report, in its 2016 Higher Education Edition [19] and 2017 K12 Edition [20], identifies VR as one of the important developments in educational technologies that are very likely to drive technology planning and decision-making over the next two or three years since the reports' date of publication. VR boosts engagement and motivation by providing students with a strong sense of presence and immersion compared to traditional experimental environment. For instance, students have the opportunity to navigate inside the human body's bloodstream as a red blood cell in The Body VR [21]. Educators, researchers, and design teams at North Carolina State University are using interactive $360^{\circ}$ video capture and VR presentation to better support lab-based and field-based instruction in online learning environments [22]. [23] presents an integrated web-based learning environment of the execution of virtual volumetric analysis experiments in chemistry with the active participation for students. VR also gets an excellent performance for learning in environments that are inherently dangerous, i.e. fire-fighting or bioterrorism situations [24]. Experiences like these provide unique and fresh moments that could hold the students' attention and pique their interest in the learning process.

More specifically, in the realm of control engineering education, taking the fundamental objectives of engineering instructional laboratories summarized in [25] into consideration, the VR interactive feature would provide an access of conducting experiment which cannot be done in the real world due to various kinds of factors, and help to reinforce the comprehending capacity of abstract control theory. Thus, it contributes to meet the objectives of Instrumentation, Models, and Experiment. And with 
strong sense of presence and immersion, it would exercise students' creativity and stimulate their senses in a realistic environment, which meets the objectives of Creativity and Sensory Awareness.

This paper integrates the VR interactivity within NCSLab for control engineering education, whose technical solution could be inspirational for other online laboratories. Section II introduces the technical implementation of VR which mainly refers to adopt Three.js as the 3D graphic engine base on Web Graphics Library (WebGL). Section III gives an overall description of integrating VR within NCSLab framework as a new interactive mode. In addition, a control engineering experiment of the ball and beam system is taken as an example to illustrate the whole operational approach of the VR experiment in Section IV. At last, Section V makes a brief summary of the study.

\section{$2 \quad$ VR Technical Solution}

The most common choice to realize VR in web-based online laboratories has been the browser plug-in for years. As a mainstream technical solution, Virtual Reality Modeling Language (VRML) which is a standard file format, has been widely adopted by online laboratories to present three-dimensional (3D) interactive vector graphics. Among the pioneer of hybrid laboratories on control engineering, the VCLab in German presented the VR realization architecture in [26] which falls back on VRML plug-in and Java Applets. The aforementioned chemical VR laboratories [23] also announced that the installation of a browser plug-in, the Cortona VRML for Parallel Graphics, is a prerequisite. The research towards a virtual radiopharmacy laboratory in Greece [27] based on the framework of VirRAD European project keeps away from VRML by using Flash Player and Shockware Player, but also failed to get rid of the dependence of browser plug-in facing the VR realization issue.

The downsides of using browser plug-in could be obviously. The application based on plug-in is often restricted to specific browser vendor, or more precisely, specific browser kernels, which naturally decrease the accessibility. In addition, the installation of plug-in is unfriendly for employment, and surely bring inconvenience for tablet/mobile users. The most important is, plug-in leads to serious security issues not only on client-side but also on server-side. Despite of the security and compatibility problems mentioned above, the developer of online laboratories have no choice but using plug-in in VR implementation in the early years.

Nowadays, as some of the plug-ins such as Java Applets are being progressively unsupported by most web browsers, new JavaScript standards (HTML5, WebGL, etc.) already provide rich interactive feature [12], and the current trend is moving to JavaScript. This paper picked WebGL as the supporting technology. WebGL is a JavaScript API used for rendering interactive 3D computer graphics directly within web browsers. It could accelerate $3 \mathrm{D}$ rendering process by using GPU, which makes it possible for running 3D application smoothly inside the modern web browsers, such as Chrome, Edge and Firefox. 
Based on WebGL, there are many 3D graphic engines available in the market such as Unity, Unreal, Three.js and Babylon.js. The technical solution of VR in this paper mainly refers to Three.js toolkit, which is a practical 3D JavaScript library.

\subsection{VR Application in 3D Graphic Engine}

Three.js core library and a few key modules need to be embedded into the head of webpage at the very beginning, whose functionalities are interpreted as in Table 1.

Table 1. Functionality of integrant module

\begin{tabular}{|l|l|}
\hline \multicolumn{1}{|c|}{ Module Name } & \multicolumn{1}{c|}{ Functionality } \\
\cline { 1 - 1 } three.js & The core library for Three.js \\
\hline DDSLoader.js & Loaders for processing external 3D model resources \\
\hline MTLLoader.js & $\begin{array}{l}\text { Split regular 3D graphics to provide the illusion of depth (an "off-axis } \\
\text { stereoscopic effect") for VR experience }\end{array}$ \\
\cline { 1 - 2 } OBJLoader.js & Provides the support for tracking the rotation of the VR display device \\
\hline StereoEffect.js &
\end{tabular}

All global variables and arguments are initialized and configured in function init. In Three.js, the Scene is the most fundamental component which works like a big container to hold every other element in. Other essential components are instantiated by constructor supplied in Three.js and appended as the child objects of the Scene. For example, Camera component is instantiated to set up viewpoint for observation, while Light component is instantiated to control the illumination intensity and direction in the virtual environment.

All the graphics captured by the Camera will be processed by the Renderer component, then projected to screen. In order to generate VR stereoscopic view, StereoEffect is instantiated by the constructor imported earlier in StereoEffect.js with the Renderer passed as the parameter. Key codes are as follows:

effect $=$ new THREE.StereoEffect $($ renderer $)$;

Then, DeviceOrientation event listener is configured by function addEventListener to be triggered when users' head rotates. Then by overwriting the callback function, which named setOrientationControls here, the viewpoint of virtual environment will follow users' behaviors synchronously. Key codes are as follows:

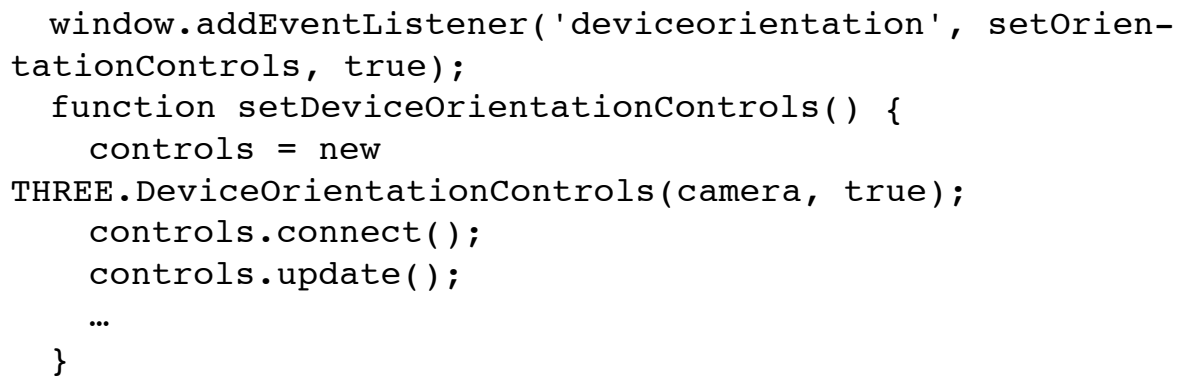


Completing the basic configuration of virtual environment, it turns to load the extern model resources. Using the related JS module such as DDSLoader.js, MTLLoader.js and OBJLoader.js, 3D models can be appended as child objects of the Scene by indicating the target file name and the path. Taking loading process of the ball and beam system as an example, the many accessories of the system such as the base, beam, axis, lever arm and ball are loaded in turn and placed at the initial position.

At the end of function init, another function named animate is called to refresh the physical state of the 3D models on each frame. And function requestAnimationFrame invokes the animate recursively. Key codes are as follows:

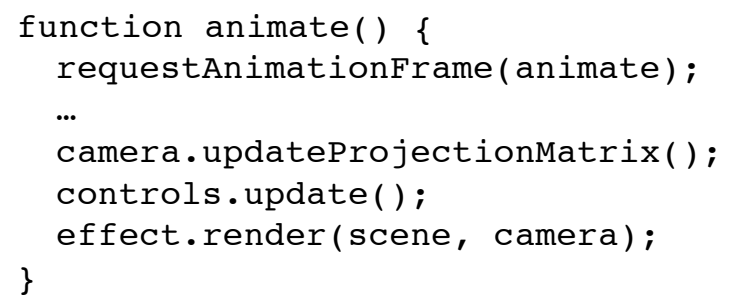

Then, VR stereoscopic animation is rendered in the web browser supported by Three.js graphic engine. On the screen of smartphone, the frame is segmented into two parts as in Fig. 1. It seems that the left part has the quite same image with the right part. Actually, subtle distinction on the angle of view finally achieves the VR visualization effect with a head-mounted display (HMD) equipped.

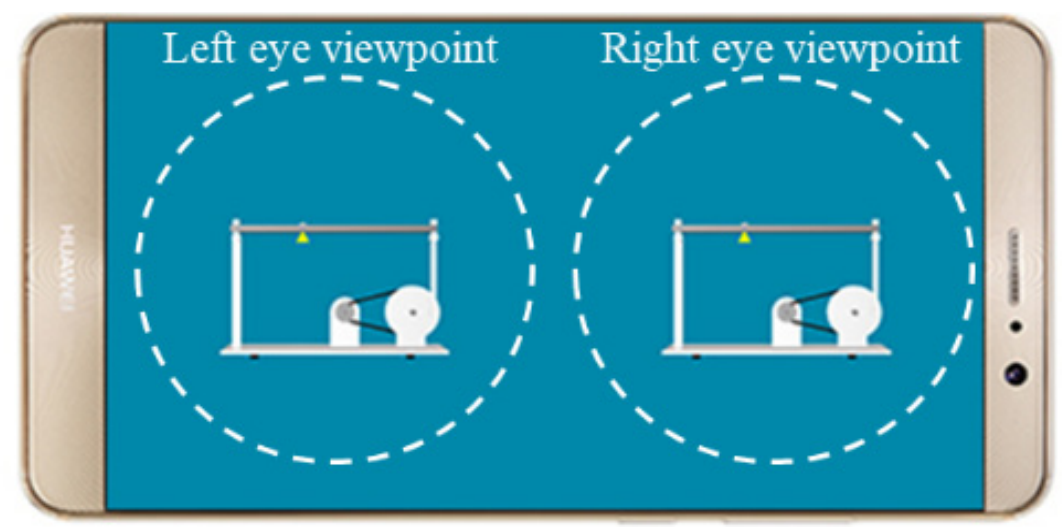

Fig. 1. VR frame on the screen of smartphone

\subsection{Auxiliary Equipment}

Generally, as shown in Fig. 2, all the HMDs on the market break down into two categories: high-end HMDs, such as the Oculus Rift, HTC Vive, or Sony PlayStation VR, and mobile-based HMDs that include the Samsung Gear VR and Google Cardboard along with accessories like headphones and haptic controller accessories. 
High-end HMDs have comfortable user experience with independent display screen, complex device structure and advanced technology. Some even have strong computation ability with powerful sensor system as an independent professional equipment. Mobile-based HMDs have a simple structure with a bargain price. It completely depends on smartphone to exhibit VR stereoscopic animation and utilizes optical structures to create depth perception.

Considering the accessibility of interest, mobile-based HMD is finally adopted by NCSLab as the auxiliary device supporting VR. Thus, users won't need to spend on costly VR HMDs. A smartphone and a mobile-based HMD is enough for users to conduct a VR experiment.

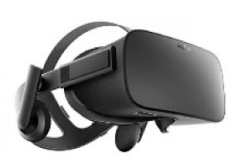

(a) Oculus Rift

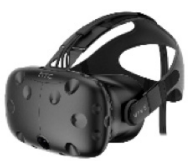

(b) HTC Vive

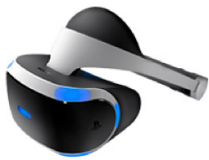

(c) PlayStation VR

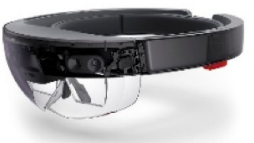

(d) HoloLens

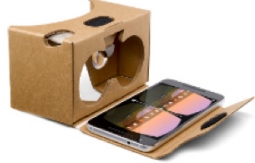

(e) Google Cardboard

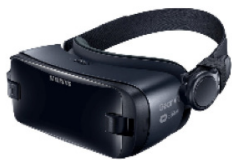

(f) Samsung Gear VR

Fig. 2. Various kinds of HMDs

\section{VR Interactive Feature of NCSLab}

NCSLab is a solid and mature hybrid laboratory framework in the realm of control engineering education. After over ten years' development, it has integrated many powerful, plugin-free interactive features [13]. The VR interactive feature presented in this paper is based on the structure of original NCSLab. As shown in Fig. 3, it has a multi-tier structure which enables reliable communication channels among the web browsers, central web server, and experiment servers located dispersedly all over the world. Users can easily get the access of all the test rigs integrated in NCSLab under a universal web-based interface without considering their physical locations.

In NCSLab framework, Three.js is adopted as the 3D graphic engine to support 3D animations and VR stereoscopic animation. Based on HTML5 standard, Three.js behaves excellently in rendering interactive $3 \mathrm{D}$ graphic within any compatible web browser. Therefore, NCSLab could provide more fluid experience and more vivid animation at a faster speed. 


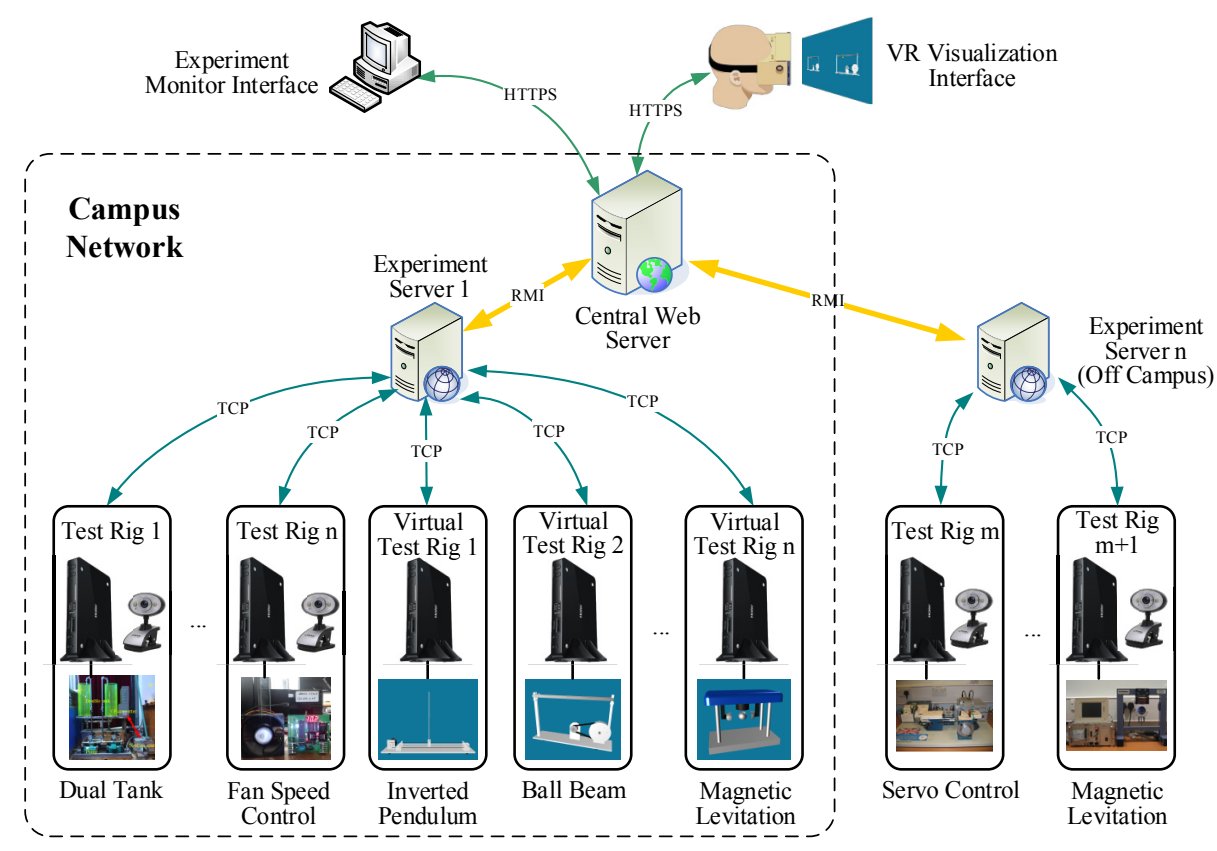

Fig. 3. Architecture of NCSLab with VR interactive feature

\subsection{D Modeling}

All the physical models such as test rigs and laboratory rooms of NCSLab must be modeled in 3D software first before imported into 3D graphic engine. Among the popular commercial software used for professional 3D designing, 3ds Max is adopted in NCSLab's 3D modeling scheme. The models designed in the 3ds Max are exported into the Wavefront.obj format which can be parsed by Three.js. Fig. 4 is an example which shows the $3 \mathrm{D}$ model of the ball and beam system being designed in $3 \mathrm{ds}$ Max environment.

During the 3D modeling process, a lot of principles must be considered seriously. On one hand, 3D models should be constructed according to the physical dimension to ensure a realistic visualization effect. On the other hand, too more non-critical modeling details will naturally lead to big size of the target files, and further cause a long download time in the internet environment. In consequence, the issues mentioned need to be balanced scrupulously. 


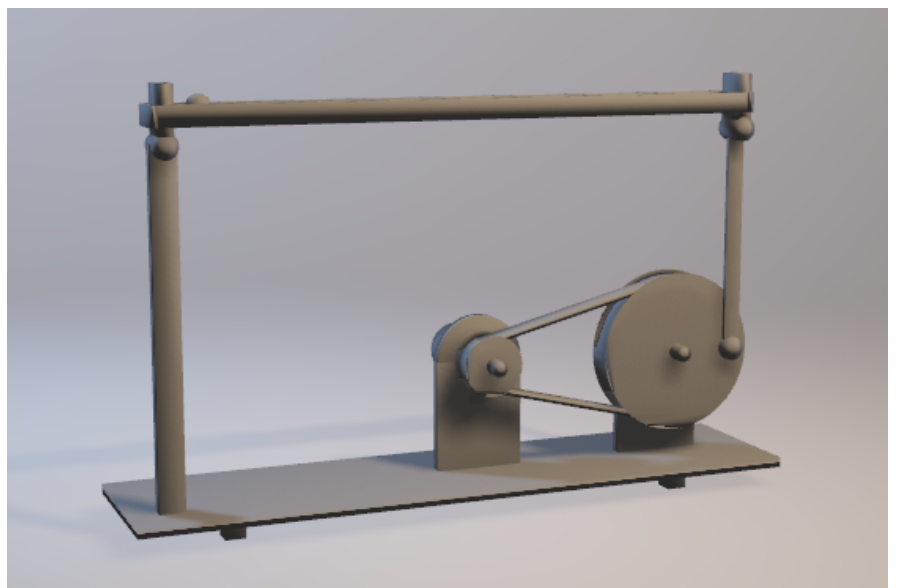

Fig. 4. Model of the ball and beam system designed in 3 ds Max

\subsection{Data Communication}

After years of debugging and modification, a mature data communication scheme among the user interface, experiment server and remote controllers finally become as shown in Fig. 5.

On the server-side, there are many Experiment Engines deployed in the Experiment Server. Each one corresponds to a test rig separately. In the Experiment Engine, three major function modules play a key role in the communication channel, which named Data Exchange Servlet, Real-Time Data Pool and Experiment Execution Module respectively. When a control algorithm is downloaded and executed in the Simulation Unit, a Transfer Control Protocol (TCP) communication is established automatically between the Experiment Execution Module and the Supervisory Control and Data Acquisition (SCADA) Server running in the Simulation Unit. The status data is captured persistently from the Simulation Unit and transmitted to the Experiment Execution Module through the TCP communication, further temporarily stored in Real-Time Data Pool.

On the client-side, after completing the configuration of monitoring interface and launching the experimental process, users can access the VR Visualization Interface in WebGL-supported mobile browser expediently by scanning a QR code. After the VR visualization web page loaded completely, a HTTPS (Hyper Text Transfer Protocol over Secure Socket Layer) communication will be established between the user interface and Data Exchange Servlet. During the whole experimental process, the Servlet continually transmits the latest status data from the Real Time Data Pool to the front-end interface. A dedicated DataStream object is designed for the correspondence of status data. It makes requests to the server at a constant frequency of twice per second, and gather the status data in buffers to ensure a continuous data stream available for Three.js graphic engine. 


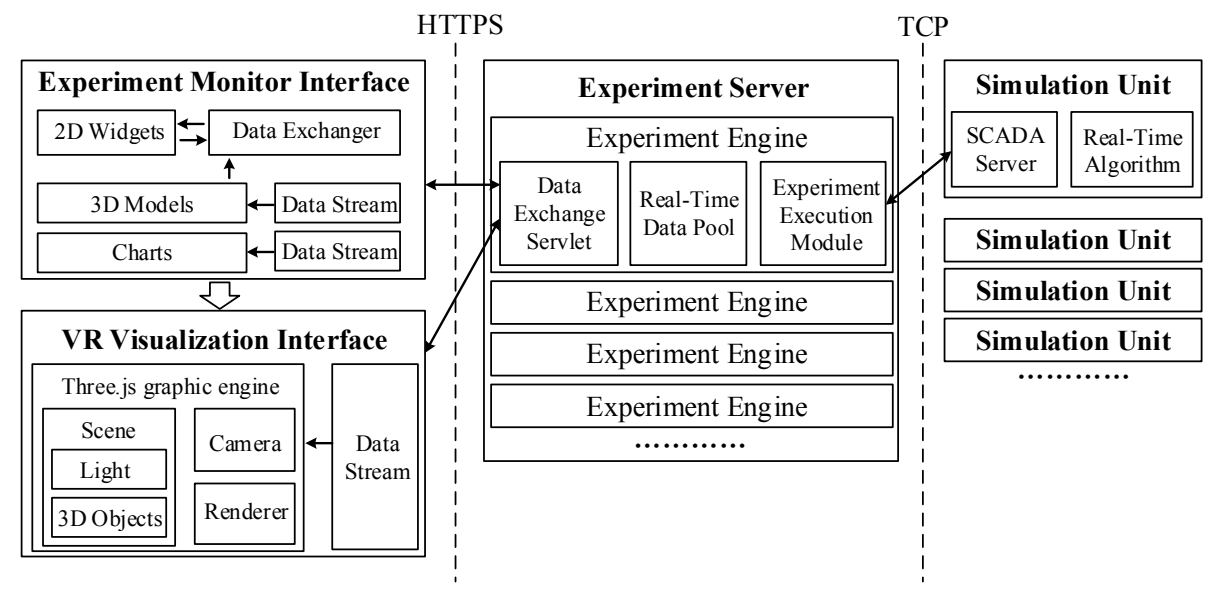

Fig. 5. Data communication schemes for VR interactivity

\subsection{VR Visualization Interface}

Three.js provides plenty of alternative JavaScript resources for customizing 3D animations as users' will. Among them, StereoEffect.js and DeviceOrientationControls.js are the foremost object which need to be embedded in the head of the webpage. More specifically, StereoEffect.js works as the most fundamental component supporting the render of VR stereoscopic animation while Device OrientationControl.js captures the rotation of VR display device.

All the latest status data of experimental process transmitted from Data Exchange Servlet are cached by the Data Stream object. Then, in the callback function animate, the data is extracted by the Three.js graphic engine and utilized for the modification of 3D models' positions and angles.

As a result, the VR stereoscopic animation keeps good consistency with remote experimental process by updating status data from datalinks. That means both of the native 3D animation and VR stereoscopic animation, one on PC desktop browser while the other on mobile browser, are synchronized with the real experiment process.

\section{$4 \quad$ A Case Study}

NCSLab has been applied in the undergraduate teaching system for years. It serves for several experimental curriculums in the field of control engineering education. According to a questionnaire implemented by the website builder, NCSLab actually plays an active role in helping students understand the course content and improve laboratory skills [13].

Now, VR Visualization Interface has been developed by the NCSLab team. The new interactive mode could enhance the sense of presence and immersion in the experimental process, thus improve the learning outcome achievement. To demonstrate 
the opinion, the ball and beam system is taken as an example to make out how to conduct a VR experiment.

Several jobs need to be managed first. Students or researchers should customize their own control algorithms based on the feature analysis of the target control system. In terms of the ball and beam system, both PD control and LQR control are proper algorithms used for making the system stable. This step need to be accomplished by Matlab Real-Time Workshop (RTW) module in the Matlab Server environment. Actually, there already have some classical control algorithms with simple construction available in the NCSLab for quick experience. After compiling the algorithm into executable program, users should upload it to the NCSLab server database through the web interface. And the executable programs will be downloaded to the Simulation Unit automatically. After that, a new window pops up for the flexible configuration of Experiment Monitor Interface. There are many kinds of 2D and 3D widgets optional for users such as dynamical charts, sliders, virtual gauges, numeric inputs.

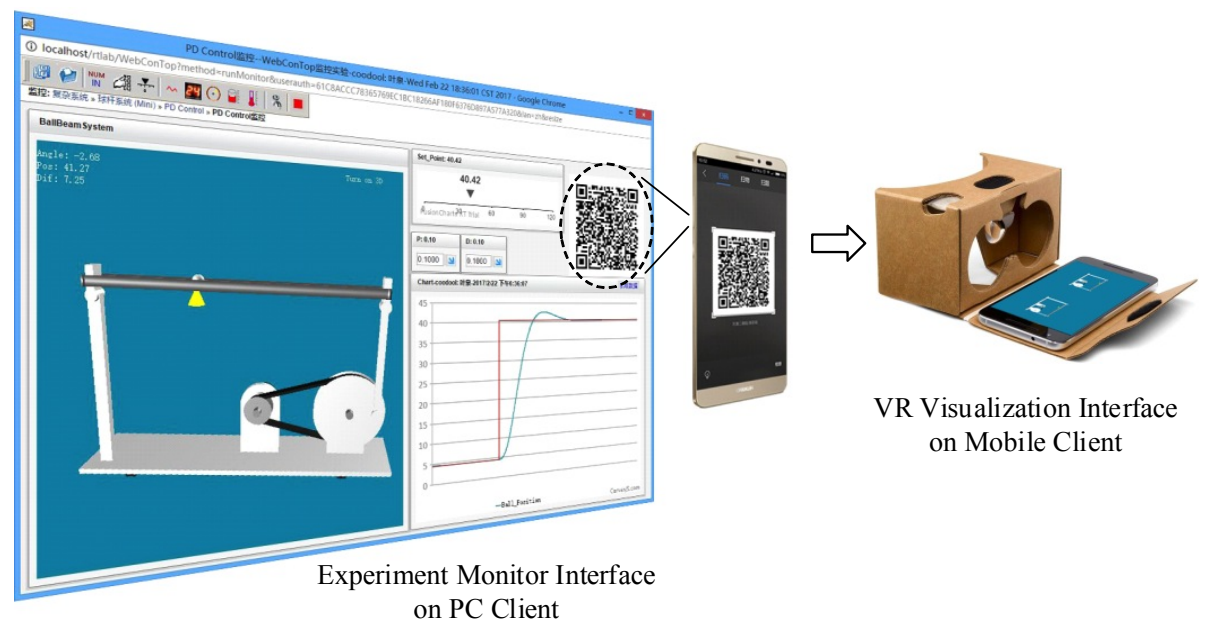

Fig. 6. Get the access of VR Visualization Interface by scanning QR code

While the preparatory work completed, online experiment is ready for launching. As in Fig. 6, by scanning the dynamic generated QR code on the top right of the Experiment Monitor Interface, users could get the only access of VR Visualization Interface. Through extracting the latest status data from the datalinks, the position of the ball and the angle of the beam in VR stereoscopic animation are synchronized with the remote experimental process as well as the 3D animations in Experiment Monitor Interface.

In a practical application scenario, all the teachers' interactive manipulations in Experiment Monitor Interface, such as changing control parameters, shifting viewpoint, could be reflected in the VR Visualization Interface with synchronous animation. As in Fig. 7, with HMD equipped, students could experience the realistic and immersive VR experiment just like they are doing a real experiment. Furthermore, 
students also have access to transform the viewpoint by rotating their heads, which will be tracked by the gyroscope sensor of smartphone and further captured by the Three.js graphic engine.

With this new interactive feature applied in the control engineering education, students are expected to achieve the following learning outcomes:

1. The ability to conduct a hands-on experiment in the real world independently;

2. The grasp of the basic working theory of test rigs;

3. Accurate comprehension of control parameters for automatic control system;

4. The ability to assemble appropriate control parameters to make control system stable or meet other specific needs;

5. The ability to identify the origin of the abnormal outcome of the control system and then re-engineer effective solutions.

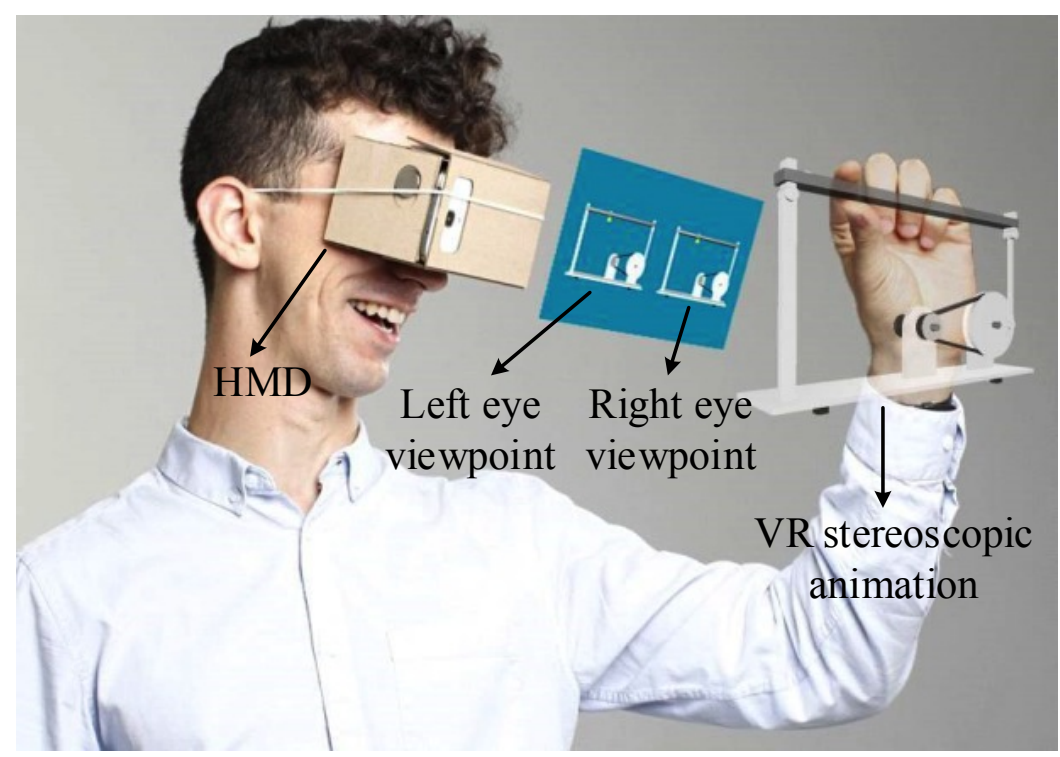

Fig. 7. Experience the VR experiment

\section{Conclusion}

This paper introduces the design and implementation of the VR interactive feature for control engineering education based on the NCSLab framework. Both the technical solution of VR stereoscopic animation using Three.js and the integration scheme on existing online laboratories framework are illustrated minutely. Compared with other online laboratories on engineering education, the VR technical solution adopted by NCSLab eliminates the dependency of plugin by keeping pace with the fastchanging modern standards and technologies, thus increasing the accessibility and availability of NCSLab itself. In addition, the ball and beam system is taken as an 
example to demonstrate the process of conducting a VR experiment. With HMD equipped, students or researchers could get an authentic and immersive VR experience which beyond the traditional online laboratories' comparison. The new VR interactive feature of NCSLab could lead to the increase of students' engagement and motivation with a strong sense of presence and immersion and provide an arena for visualizing abstract concepts concretely, and the plugin-free technical solution could be inspirational for other web-based online laboratories to integrate such a new interactive feature.

\section{Acknowledgment}

This research is supported by National Natural Science Foundation (NNSF) of China under Grant 61374064.

\section{$7 \quad$ References}

[1] R. Heradio, L. De Torre, and S. Dormido, "Virtual and remote labs in control education: A survey,” Annu. Rev. Control, vol. 42, pp. 1-10, 2016. https://doi.org/10.1016/j.arcontrol. 2016.08.001

[2] V. Potkonjak et al., "Virtual laboratories for education in science, technology, and engineering: A review," Comput. Educ., vol. 95, pp. 309-327, 2016. https://doi.org/10.1016/j.compedu.2016.02.002

[3] L. Gomes and S. Bogosyan, "Current Trends in Remote Laboratories," IEEE Trans. Ind. Electron., vol. 56, no. 12, pp. 4744-4756, Dec. 2009. https://doi.org/10.1109/ TIE.2009.2033293

[4] C. Gravier, J. Fayolle, B. Bayard, M. Ates, and J. Lardon, "State of the art about remote laboratories paradigms-foundations of ongoing mutations," Int. J. Online Eng., vol. 4, no. 1, pp. 19-25, 2008.

[5] Z. C. Zacharia, "Comparing and combining real and virtual experimentation: an effort to enhance students' conceptual understanding of electric circuits," J. Comput. Assist. Learn., vol. 23, no. 2, pp. 120-132, Jan. 2007. https://doi.org/10.1111/j.1365-2729.2006.00215.x

[6] T. de Jong, M. C. Linn, and Z. C. Zacharia, "Physical and Virtual Laboratories in Science and Engineering Education,” Science (80-. )., vol. 340, no. 6130, pp. 305-308, Apr. 2013.

[7] J. R. Brinson, "Learning outcome achievement in non-traditional (virtual and remote) versus traditional (hands-on) laboratories: A review of the empirical research," Comput. Educ., vol. 87, pp. 218-237, Sep. 2015. https://doi.org/10.1016/j.compedu.2015.07.003

[8] W. Hu, G. P. Liu, D. Rees, and Y. Qiao, "Design and implementation of web-based control laboratory for test rigs in geographically diverse locations," IEEE Trans. Ind. Electron., vol. 55, no. 6, pp. 2343-2354, 2008. https://doi.org/10.1109/TIE.2008.920646

[9] Y. Qiao, G. P. Liu, G. Zheng, and W. Hu, "NCSLab: A web-based global-scale control laboratory with rich interactive features," IEEE Trans. Ind. Electron., vol. 57, no. 10, pp. 3253-3265, 2010. https://doi.org/10.1109/TIE.2009.2027924

[10] W. Hu, G. P. Liu, and H. Zhou, "Web-based 3-D control laboratory for remote real-time experimentation," IEEE Trans. Ind. Electron., vol. 60, no. 10, pp. 4673-4682, 2013. https://doi.org/10.1109/TIE.2012.2208440 
Paper-VR Interactive Feature of HTML5-based WebVR Control Laboratory by Using Head-mount...

[11] W. Hu, H. Zhou, Z. W. Liu, and L. Zhong, "Web-based 3D interactive virtual control laboratory based on NCSLab framework," Int. J. Online Eng., vol. 10, no. 6, pp. 10-18, 2014. https://doi.org/10.3991/ijoe.v10i6.3845

[12] Z. Lei, W. Hu, and H. Zhou, "Deployment of a Web-based Control Laboratory Using HTML5," Int. J. Online Eng., vol. 12, no. 7, pp. 18-23, 2016. https://doi.org/10.3991/ ijoe.v12i07.5819

[13] W. Hu et al., "Plug-in free web-based 3-D interactive laboratory for control engineering education," IEEE Trans. Ind. Electron., vol. 64, no. 5, pp. 3808-3818, 2017. https://doi.org/10.1109/TIE.2016.2645141

[14] Z. Pan, A. D. Cheok, H. Yang, J. Zhu, and J. Shi, "Virtual reality and mixed reality for virtual learning environments," Comput. Graph., vol. 30, no. 1, pp. 20-28, 2006. https://doi.org/10.1016/j.cag.2005.10.004

[15] B. Dalgarno and M. J. W. Lee, "What are the learning affordances of 3-D virtual environments?," Br. J. Educ. Technol., vol. 41, no. 1, pp. 10-32, 2010. https://doi.org/10.1111/j.1467-8535.2009.01038.x

[16] G. Burdea and P. Coiffete, Virtual Reality Technology. John Wiley \& Sons, 2003.

[17] Z. Merchant, E. T. Goetz, L. Cifuentes, W. Keeney-Kennicutt, and T. J. Davis, "Effectiveness of virtual reality-based instruction on students' learning outcomes in K-12 and higher education: A meta-analysis," Comput. Educ., vol. 70, pp. 29-40, 2014. https://doi.org/10.1016/j.compedu.2013.07.033

[18] C. J. Chen, S. C. Toh, and W. M. F. W. Ismail, "Are Learning Styles Relevant To Virtual Reality?," J. Res. Technol. Educ., vol. 38, no. 2, pp. 123-141, Dec. 2005. https://doi.org/10.1080/15391523.2005.10782453

[19] L. Johnson, S. Adams Becker, M. Cummins, A. Freeman, and C. Hall, "NMC horizon report: 2016 higher education edition,” Austin, Texas: The New Media Consortium, 2016.

[20] A. Freeman, S. Adams Becker, M. Cummins, A. Davis, and C. Hall Giesinger, "NMC/CoSN Horizon Report: 2017 K-12 Edition," Austin, Texas: The New Media Consortium, 2017.

[21] "The Body VR.” [Online]. Available: http://thebodyvr.com/.

[22] " $360^{\circ}$ Video, Virtual Reality Presentation Highlights Edu Applications." [Online]. Available: https://delta.ncsu.edu/news/2015/12/01/360o-video-virtual-reality-presentationhighlights-edu-applications/.

[23] J. Georgiou, K. Dimitropoulos, and a. Manitsaris, "A virtual reality laboratory for distance education in chemistry," World Acad. Sci. Eng. Technol., vol. 1, no. 11, pp. 345-352, 2007.

[24] J. N. Bailenson, N. Yee, J. Blascovich, A. C. Beall, N. Lundblad, and M. Jin, "The Use of Immersive Virtual Reality in the Learning Sciences: Digital Transformations of Teachers, Students, and Social Context," J. Learn. Sci., vol. 17, no. 1, pp. 102-141, Feb. 2008. https://doi.org/10.1080/10508400701793141

[25] L. D. Feisel and A. J. Rosa, "The Role of the Laboratory in Undergraduate Engineering Education," J. Eng. Educ., vol. 94, no. 1, pp. 121-130, Jan. 2005. https://doi.org/10.1002/ j.2168-9830.2005.tb00833.x

[26] C. Schmid, "Virtual control laboratories and remote experimentation in control engineering," in Proc. 11th EAEEIE Annual Conference on Innovations in Education for Electrical and Information Engineering, University of Ulm, Ulm, 2000, pp. 213-218.

[27] A. Alexiou, C. Bouras, E. Giannaka, V. Kapoulas, M. Nani, and T. Tsiatsos, "Using VR technology to support e-learning: The 3D virtual radiopharmacy laboratory," 24th Int. Conf. Distrib. Comput. Syst. Work. Proc., pp. 268-273, 2004. https://doi.org/10.1109/ ICDCSW.2004.1284042 


\section{Authors}

Quan Ye received the B.S. degree in automation from Wuhan University, Wuhan, China, in 2011, where he is now working towards the M.Sc. degree in the Department of Automation. His current research interests include networked control systems and web-based remote laboratories.

Wenshan Hu received the B.S. and M.Sc. degrees in control theory and applications from Wuhan University, Wuhan, China, in 2002 and 2004, respectively, and the $\mathrm{Ph} . \mathrm{D}$. degree in control engineering from the University of Glamorgan, Pontypridd, U.K. in 2008. In June 2010, he joined Wuhan University as an Associate Professor. He has been a professor since 2016. His research interests include network-based control laboratories and wireless power transfer. (e-mail: wenshan.hu@whu.edu.cn)

Hong Zhou received the B.S. degree in automation from the Central South University of Technology, Changsha, China, in 1982, the M.Sc. degree in control theory and applications from Chongqing University, Chongqing, China, in 1988, and the $\mathrm{Ph}$.D. degree in mechanical engineering from Wuhan University, Wuhan, China, in 2006. He has been a professor in the Department of Automation, Wuhan University, since 2000. His research interests include wireless power transfer, smart grid, and networked control systems.

Zhongcheng Lei received the B.S. degree in automation from Wuhan University, Wuhan, China, in 2014, where he is currently working toward the Ph.D. degree in the Department of Automation. His current research interests include networked control systems and web-based remote laboratories.

Shiqi Guan received the B.S. degree in automation from Wuhan University of Technology, Wuhan, China, in 2012. She is now working towards the M.Sc. degree in Department of Automation, Wuhan University. Her current research interests include networked control systems and web-based remote laboratories.

Article submitted 13 December 2017. Resubmitted 02 February 2018. Final acceptance 05 March 2018. Final version published as submitted by the authors. 